\title{
Deletion of SLC19A2, the High Affinity Thiamine Transporter, Causes Selective Inner Hair Cell Loss and an Auditory Neuropathy Phenotype
}

\author{
M. C. Liberman, ${ }^{1,2}$ E. Tartaglini, ${ }^{3}$ J. C. Fleming, ${ }^{3}$ and E. J. Neufeld ${ }^{3}$ \\ ${ }^{1}$ Eaton-Peabody Laboratory, Massachusetts Eye and Ear Infirmary, 243 Charles Street, Boston, MA 02114, USA \\ ${ }^{2}$ Department of Otology and Laryngology, Harvard Medical School, Boston, MA 02114, USA \\ ${ }^{3}$ Division of Hematology, Children's Hospital, Boston, MA, USA
}

Received: 21 February 2006; Accepted: 24 March 2006; Online publication: 27 April 2006

\begin{abstract}
Mutations in the gene coding for the high-affinity thiamine transporter Slc19a2 underlie the clinical syndrome known as thiamine-responsive megaloblastic anemia (TRMA) characterized by anemia, diabetes, and sensorineural hearing loss. To create a mouse model of this disease, a mutant line was created with targeted disruption of the gene. Cochlear function is normal in these mutants when maintained on a high-thiamine diet. When challenged with a low-thiamine diet, Slc19a2-null mice showed 40-60 dB threshold elevations by auditory brainstem response (ABR), but only 10-20 dB elevation by otoacoustic emission (OAE) measures. Wild-type mice retain normal hearing on either diet. Cochlear histological analysis showed a pattern uncommon for sensorineural hearing loss: selective loss of inner hair cells after 1-2 weeks on low thiamine and significantly greater inner than outer hair cell loss after longer low-thiamine challenges. Such a pattern is consistent with the observed discrepancy between ABR and OAE threshold shifts. The possible role of thiamine transport in other reported cases of selective inner hair cell loss is considered.
\end{abstract}

Keywords: sensorineural hearing loss, thiamine responsive megaloblastic anemia

Correspondence to: M. C. Liberman · Eaton-Peabody Laboratory, Massachusetts Eye and Ear Infirmary · 243 Charles Street, Boston, MA 02114, USA. Telephone: (1)617-5733745; fax: (1)617-7204408; email: charles_liberman@meei.harvard.edu

\section{INTRODUCTION}

Thiamine pyrophosphate, also known as vitamin B1, is a coenzyme required for several key steps in intermediary metabolism, including the oxidative decarboxylation of pyruvate and $\alpha$-ketoglutarate. Most cells require these enzymes for energy production via the Krebs cycle. Although plentiful in foods, there is little synthesis by intestinal microorganisms, thus symptoms (e.g., beriberi) appear rapidly after dietary deprivation.

Thiamine-responsive megaloblastic anemia (TRMA) syndrome is an early-onset, autosomal recessive disorder also characterized by diabetes and sensorineural hearing loss. The gene responsible (SLC19A2) encodes a high-affinity thiamine transporter (Diaz et al. 1999; Fleming et al. 1999; Labay et al. 1999). Within the cochlea, this high-affinity transporter is expressed in inner hair cells (IHC) (Fleming et al. 2001).

To further study the disease, a mouse line was created with targeted disruption of the SLC19A2 gene (Fleming et al. 2003). We describe the auditory phenotype of this mouse line, as seen via auditory brainstem responses (ABRs) and by measurement of distortion product otoacoustic emissions (DPOAEs), as well as the cochlear phenotype as seen via plasticembedded sections through the temporal bone. The functional measures suggest an auditory neuropathy phenotype, i.e., one in which cochlear neural responses, as seen by ABR, are significantly more reduced than the reduction in cochlear amplifier function, as seen in the DPOAEs. This functional 


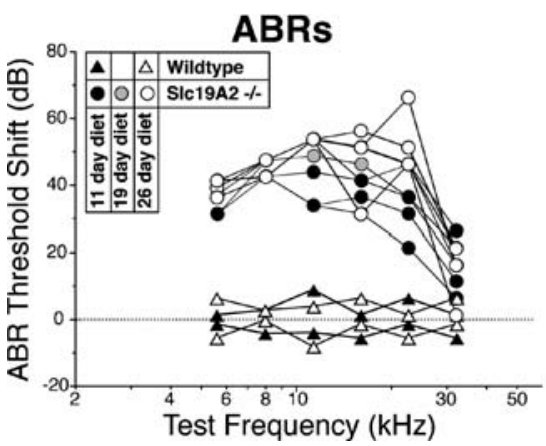

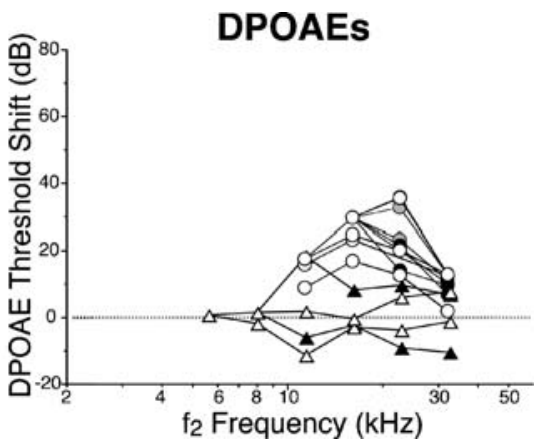

FIG. 1. Threshold shifts for wild-type and transporter-null mice placed, at age 45 days, on a low-thiamine diet for 11,19 , or 26 days, as indicated in the key. Until age 45 days, all animals in this cohort were maintained on a normal diet. Threshold shift for both groups is defined with reference to the average thresholds for wild-type ears. phenotype is associated with a histological pattern of selective IHC loss, an extremely rare type of sensorineural histopathology.

\section{METHODS}

\section{Animals and Groups}

All animals were maintained on a normal diet (22 $\mathrm{mg} / \mathrm{kg}$ thiamine) until they were $\sim 45$ days of age. Next, a subset of wild-type and homozygous mutants were put on a low-thiamine diet $(2 \mathrm{mg} / \mathrm{kg})$. After $12-$ 38 days, DPOAEs and tone pip evoked ABRs were recorded, and cochleas were harvested for histopathological analysis. In total, data were obtained from 22 transporter-null and 11 wild-type animals with roughly equal numbers of males and females from each genotype.

\section{Gene Deletion}

The targeting vector was electroporated into male 129 ES cells, injected into $\mathrm{C} 57 \mathrm{Bl} / 6$ blastocysts. Chimeras were backcrossed to129S6/SvEvTac females, thus male transgenic offspring were pure 129S6/SvEvTac. For further details, see (Fleming et al. 2003).

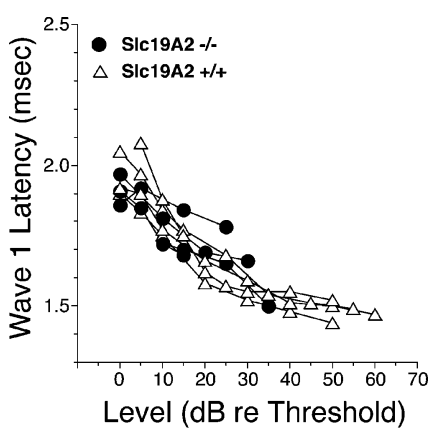

FIG. 2. Latencies for ABR wave 1 are unchanged in transporternull mice, even when thresholds are elevated by $30-40 \mathrm{~dB}$. Data are for 22.6-kHz tone pips from the cohort of animals shown in Figure 1: for transporter nulls, data are from measures after 11 days on low thiamine; for wild-types, data are from both 11 and 26 days on low thiamine.
ABRs

Mice were anesthetized with xylazine $(20 \mathrm{mg} / \mathrm{kg}$, i.p. $)$ and ketamine $(100 \mathrm{mg} / \mathrm{kg}$, i.p.). Needle electrodes were inserted at vertex and pinna. ABRs were evoked with 5-ms tone pips (0.5-ms rise-fall with a $\cos ^{2}$ onset envelope, delivered at 35 per second). The response was amplified, filtered, and averaged with an A-D board in a LabVIEW-driven data acquisition system. Sound level was raised in 5-dB steps from $10 \mathrm{~dB}$ below threshold up to $90 \mathrm{~dB}$ sound pressure level (SPL). Thresholds were determined by visual inspection of the stacked waveforms.

\section{DPOAEs}

The DPOAE at $2 f_{1}-f_{2}$ was recorded in response to primary tones: $f_{1}$ and $f_{2}$, with $f_{2} / f_{1}=1.2$ and the level of $f_{2}$ at $10 \mathrm{~dB}$ below the level of $f_{1}$. Ear-canal sound pressure was amplified and digitally sampled at $4 \mu \mathrm{s}$. Fast-Fourier transforms were computed from averaged sound pressure waveforms, and the DPOAE and surrounding noise floor were extracted. Iso-response contours were interpolated from plots of amplitude vs. sound level, performed in 5-dB steps of $f_{1}$ level. "Threshold" is defined as the $f_{2}$ level required to produce a DPOAE at $0 \mathrm{~dB}$ SPL.

\section{Histological Preparation}

Animals were perfused intracardially with $2.5 \%$ glutaraldehyde and $1.5 \%$ paraformaldehyde in phosphate buffer. Temporal bones were extracted, and round and oval windows opened for intralabyrinthine perfusion of fixative. Cochleas were then osmicated $(1 \% \mathrm{OsO} 4$ in $\left.\mathrm{dH}_{2} \mathrm{O}\right)$, decalcified $(0.1 \mathrm{M}$ EDTA with $0.4 \%$ glutaradehyde), dehydrated in ethanols and propylene oxide, embedded in Araldite resins, and sectioned at $40 \mu \mathrm{m}$ on a Historange with a carbide steel knife. Sections were mounted on slides and coverslipped.

\section{Cytocochleograms}

Each cochlear spiral was reconstructed in 3D, using Neurolucida software, and distance from the base 


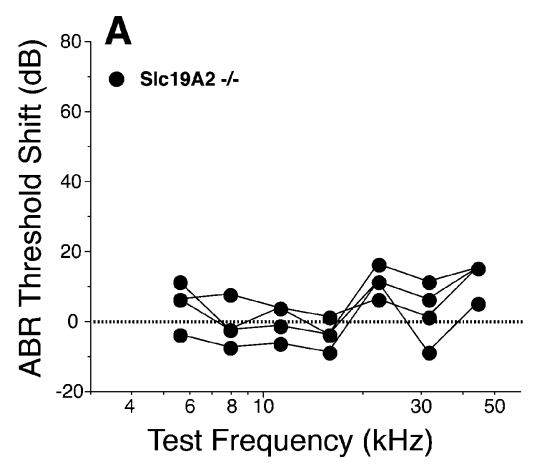

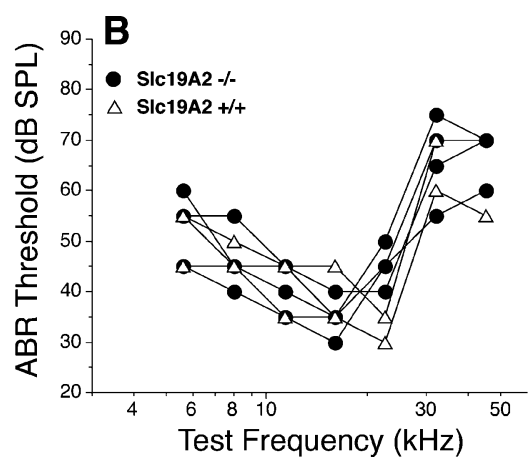

FIG. 3. Transporter-null mice maintained on a diet with normal thiamine levels showed no threshold shifts. (A) ABR data from transporternull animals plotted as threshold shifts; (B) ABR data replotted as absolute thresholds and compared to the wild-type data from Figure 1. ABR data from transporter-nulls are from mice maintained on a normal diet until 106 days of age. computed for each section through the duct. A cytocochleogram was prepared, using high-power Nomarski optics, based on the assessment of all present and absent hair cells in all sections. Cochlear location was converted to frequency according to a cochlear map for mouse (Muller et al. 2005).

\section{RESULTS}

Mice were placed on a low-thiamine diet (2 vs. $22 \mathrm{mg}$ / $\mathrm{kg}$ in normal chow) for 11, 19, or 26 days. By 11 days, cochlear neural output was significantly reduced in transporter-null mice, as seen in the threshold shifts for auditory brainstem responses (ABRs), especially for frequencies $<22 \mathrm{kHz}$ (Fig. 1). There was little further threshold shift after 19 or 26 days on low thiamine. Wild-type mice on the same low-thiamine diet showed no threshold elevation, even after 26 days (Fig. 1). Shifts in distortion product otoacoustic emissions (DPOAE) thresholds were also larger in transporter-null mice than in wild-types (Fig. 1). However, in the transporter-null mice the DPOAE shifts were smaller than the shifts in ABR thresholds ( $p=0.003, F=86.634$, for group differences by two-way ANOVA). Compare, for example, the ABR threshold shifts of $\sim 50 \mathrm{~dB}$ at 11.3 and $16 \mathrm{kHz}$ in the transporter null mice after 26 days on low-thiamine to the DPOAE shifts measured at the same time, which were only 10-20 dB. This mismatch between ABR and DPOAE threshold shifts suggests that a significant component of the cochlear dysfunction in the null mice arises at, or "downstream" of, the inner hair cell (e.g., Liberman et al. 1997).

To assess effects on the auditory nerve fibers per se, we measured wave 1 latencies from the ABRs in Figure 1. When latencies are plotted in $\mathrm{dB}$ re $\mathrm{ABR}$ threshold, there are no significant differences between wild-type and transporter-null animals at 11 days after thiamine restriction, a time when ABR thresholds are already elevated by as much as $40 \mathrm{~dB}$ (Fig. 2).

When another group of transporter-null and wildtype mice were placed on a low-thiamine diet with 3 $\mathrm{mg} / \mathrm{kg}$ thiamine (rather then $2 \mathrm{mg} / \mathrm{kg}$ used for first cohort) no significant threshold differences were observed, by either ABR or DPOAE measures, even after 70 days on the diet (data not shown). Similarly, when a third group of transporter-null mice was maintained on a diet with normal thiamine levels, no threshold shifts were seen (Fig. 3; differences between wild-type and transporter-null thresholds were not significant: $p=0.5, F=0.505$, two-way ANOVA).

A final cohort of wild-type and transporter null animals was placed on $2 \mathrm{mg} / \mathrm{kg}$ thiamine at age 45 days and then returned to a normal thiamine diet after 19 days, to see if the threshold shifts could be "rescued." As shown in Figure 4, the initial threshold shift in the transporter null animals seen after 19 days on low thiamine was, on average, $20 \mathrm{~dB}$ at the lower frequencies. There was a slight improvement in average thresholds over the next 5 weeks; however, thresholds did not return to normal levels. Variability was high in this group, because one of the null animals showed minimal threshold shift at 19 days (differences between "initial" and "rescued" thresholds were not significant: $p=0.089, F=6.193$, two-way ANOVA).

Cochleas were plastic-embedded to allow the highresolution evaluation of all cochlear structures at the

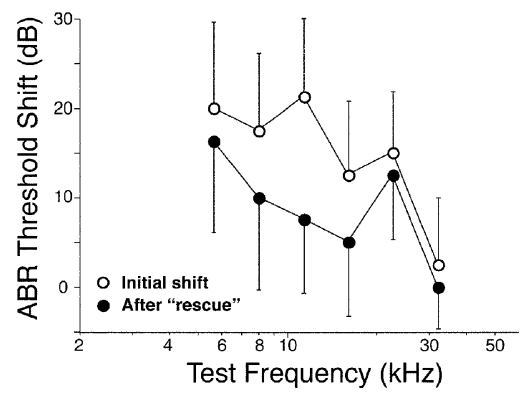

FIG. 4. Partial recovery in a rescue experiment in which transporter-null mice were maintained on a low-thiamine diet for 19 days (initial shift), and then switched back to normal diet for 35 days. For the first 45 days of life, these animals were on normal thiamine. Data points show mean for four transporter-null animals (error bars are SEMs). 

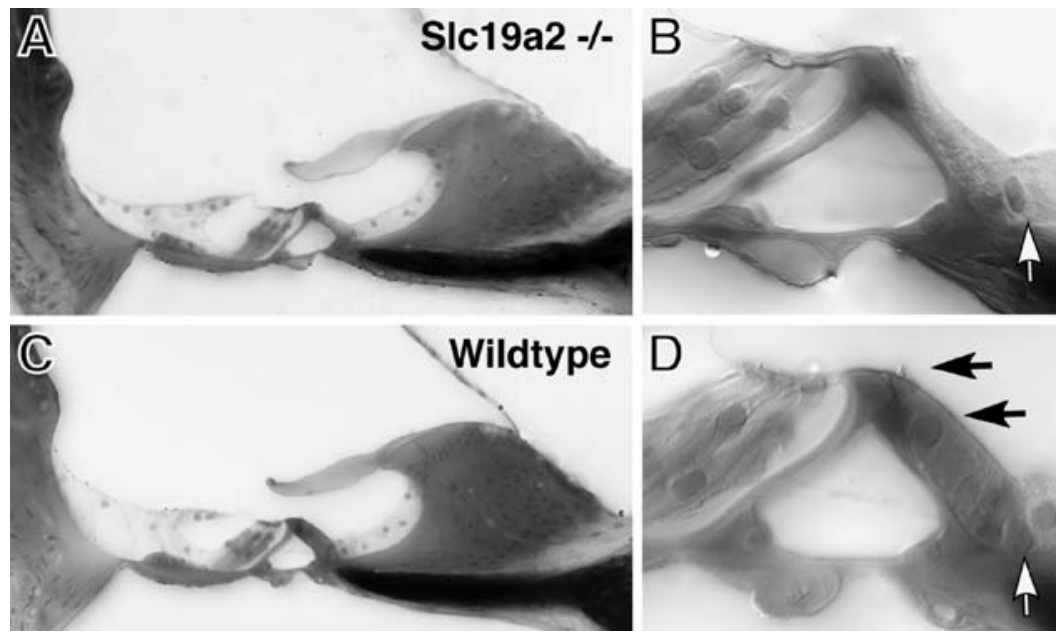

FIG. 5. When transporter-null mice were maintained on a low-thiamine diet until ABR thresholds began to shift, their cochleas showed selective loss of inner hair cells. (A, C) Place-matched images from the upper basal turn of a transporter-null and a wild-type mouse, respectively, each of which was placed on a low-thiamine diet for 26 days, beginning at 45 days of age. (B, D) contain higher-power views of the inner hair cell areas from $(A)$ and $(C)$, respectively: black arrows in (D) show the nucleus and stereocilia bundle of the normal inner hair cell; white arrows point to the nuclei of the supporting cells in the inner hair cell area that remain in both ears.

light microscopic level. The most striking histopathology in the transporter-null mice maintained on low thiamine was selective loss of inner hair cells (Fig. 5). The overall architecture of the organ of Corti remained remarkably normal; thus inspection with low-power objectives might fail to reveal the pathology. In the high-power view (insets), the nucleus and stereocilia of a normal inner hair cell are clearly visible in the wild-type (filled arrows) and clearly absent in the transporter null ear. Supporting cells in the inner hair cell area are present in both cases (unfilled arrows point to their nuclei). There was no sign of spiral ganglion cell loss, nor was there an obvious loss of peripheral axons in the osseous spiral lamina; however, the longest postdiet-onset survival was only 36 days. The stria vascularis, spiral ligament, and spiral limbus all looked normal in transporternull mice.

Cytocochleograms prepared from mice in the cohort in Figure 1 reveal a regular progression of hair cell loss with increasing duration of dietary thiamine restriction (Fig. 6). Hair cell loss is minimal in wild-type animals (Fig. 6A) and in transporter null mice sacrificed after 11 days on low thiamine (Fig. $6 \mathrm{~B})$, although ABR shifts were visible at this time in the latter group. By 19 days on low thiamine, the transporter-null mice show loss restricted to the inner hair cells in the apical half of the cochlea (Fig. 6C). By 26 days, inner hair cell loss was virtually complete throughout a large midcochlear region; however, the basal $1 / 3$ of the cochlea showed no cell loss (Fig. 6D). In the animals maintained on the diet for 36 days, inner hair cell loss had spread slightly toward the basal cochlear extreme; however, there was more extensive loss of outer hair cells $(\mathrm{OHC})$ as well (Fig. 6E).

The time progression of hair cell loss is explicitly plotted in Figure 7, in which the fractional survival of inner or outer hair cells is averaged over the apical 2/ 3 of the cochlea. The data suggest that loss of inner hair cells dramatically increases in transporter-null mice between 19 and 26 days after the onset of lowthiamine diet, and that OHC loss progresses at a significantly slower rate.

\section{DISCUSSION}

Auditory phenotype in the Slc19a2-null mouse

Our results show that the high-affinity thiamine transporter coded for by the SLC19A2 gene is necessary for cochlear function, but only when thiamine intake is restricted. In transporter-null mice, cochlear threshold shifts appeared within 11 days after thiamine was reduced to $2 \mathrm{mg} / \mathrm{kg}$ (normal mouse chow has $22 \mathrm{mg} / \mathrm{kg}$ thiamine). No threshold shifts were measurable up to 60 days after restriction of dietary thiamine to $3 \mathrm{mg} / \mathrm{kg}$. Lower thiamine doses resulted in premature death, consistent with the observation that the high-affinity thiamine transporter is widely expressed throughout the body, including erythrocytes, liver, kidney, and brain (Fleming et al. 2001). No threshold shifts were seen in wild-type mice maintained on this extremely low thiamine diet, suggesting that thiamine deficiency in the general population is not likely to be associated with an increased risk of sensorineural hearing loss.

In the cochlea, this high-affinity thiamine transporter is expressed more strongly in inner hair cells than in outer hair cells (Fleming et al. 2001). The present results suggest that it is most critical for inner hair cell function, given that inner hair cell loss appeared earlier and was always more severe than outer hair cell loss. The enhanced vulnerability of inner hair cells suggests that the other thiamine 

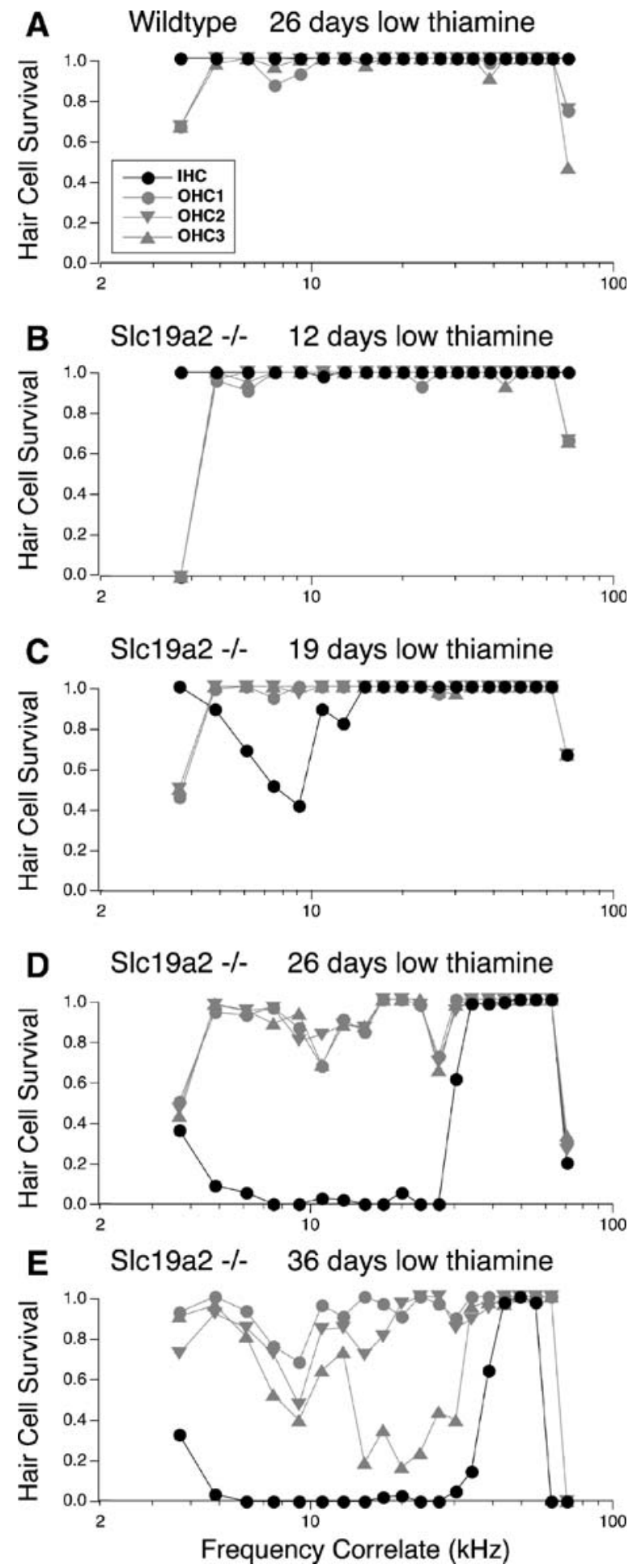

FIG. 6. Patterns of hair cell loss in representative mice from the cohort in Figure 1: i.e., animals maintained on a low-thiamine diet for variable periods (see header to each panel). (A) Data from a wildtype ear, (B-E) data from four transporter-null ears. Hair cell survival is plotted as a fraction: a value of 1.0 means that all hair cells were present. Data in each case are averaged over bins corresponding to $5 \%$ of total cochlear length. Cochlear position is translated into frequency according to a map for the mouse cochlea (Muller et al. 2005). Symbol key in (A) applies to all panels. transporter, SLC19A3 (which remains present in these Slc19a2-null mice), may also be expressed in outer hair cells. The fact that this remarkably selective hair cell loss can be externally triggered simply by changing the dietary thiamine intake makes it potentially useful in a number of experimental models, e.g., transplantation-based approaches to attempting to repopulate the damaged inner ear. Reasons for the enhanced vulnerability of apical inner hair cells in the transporter-null animals are unclear, but longitudinal gradients in the expression pattern of SLC19A2 have not been investigated.

Oishi et al. (2002) recently studied an independently derived mouse line with targeted deletion of the same SLC19A2 gene. The published ABR data are not inconsistent with the present results; the ABR threshold shifts they report are comparable (40-60 $\mathrm{dB})$ to those reported here; however, the DPOAEs were not evaluated. Surprisingly, the previous study reported no consistent histopathological change. Reasons for this discrepancy are not obvious, as the gene targeting strategies in the two lines were similar. The degree of inner hair cell loss clearly depends on the duration and magnitude of thiamine deprivation. Thus, on a different diet regimen, IHC dysfunction might be sufficient to raise ABR thresholds without the frank cell loss seen here. Although the animals in the other study were maintained on a lower level of thiamine $(0 \mathrm{mg} / \mathrm{kg})$ it is not clear how long the lowthiamine challenge lasted. Although the animals were tested at 14-16 weeks of age, the challenge must have been shorter because these authors stated that transporter null mice died after 3-4 weeks on a zero-thiamine diet (as has been the experience with our mouse line). It is also possible that inner hair cell loss occurred but was undetected in the previous study, either because evaluation was limited to the basal turn, where the loss is less complete, or simply

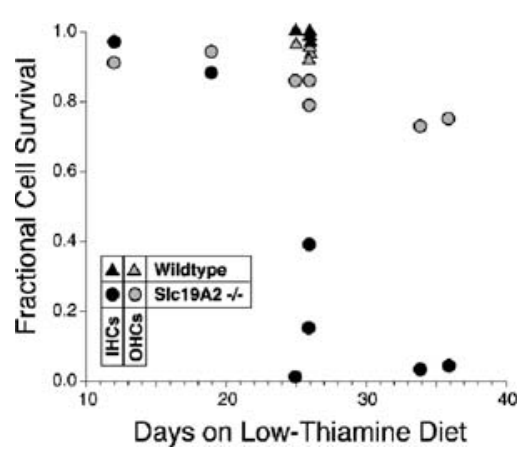

FIG. 7. Loss of inner and outer hair cells in wild-type and transporter null ears, as a function of days on low-thiamine diet. All animals were placed on the diet at 45 days of age after initial maintenance on a normal diet. Each point represents data from a different ear, averaged across all cochlear locations. Outer hair cell values represent average of data from the first-row cells only. 
because selective inner hair cell loss is such a rare occurrence coupled with the fact that the normal architecture of the organ of Corti is perfectly maintained despite the loss of inner hair cells. Thus the general tissue degradation and organ of Corti collapse that accompanies paraffin embedding, as used in the previous study, might have obscured the selective loss of inner hair cells clearly seen in plasticembedded material. Indeed, a recent preliminary report reanalyzing the cochlear phenotype in this independently generated transporter null mouse now describes selective IHC loss after low-thiamine challenge (Bertram et al. 2006).

\section{Causes of selective inner hair cell loss: a common mechanism?}

The selective loss of inner hair cells (Figs. 5 and 6) and the 20- to 30-dB mismatch between ABR and DPOAE threshold shifts (Fig. 1) suggest that disruption of the SLC19A2 gene may underlie some forms of "auditory neuropathy," a clinical diagnosis characterized by persistence of measurable OAEs in cases with profound reduction in ABRs (Starr et al. 1996). Although DPOAEs were slightly reduced in our transporter-null mice (Fig. 1), DPOAE shifts should exactly match ABR shifts when hearing loss arises from pure OHC dysfunction, as in a mutant mouse with targeted deletion of prestin, a key component of the outer hair cell molecular motor (Liberman et al. 2002). It is not clear whether hearing loss in patients with thiamine-responsive megaloblastic anemia (TRMA), the heritable disease arising from mutations in the SLC19A2 gene deleted in our mice, would be characterized as an auditory neuropathy; appropriate clinical data have not been published.

Selective loss of inner hair cells is an extremely rare type of cochlear histopathology (Schuknecht 1974). In most cases of sensorineural hearing loss, whether due to noise (Liberman and Kiang 1978), ototoxic drugs (Huizing and de Groot 1987), or genetic anomalies, the loss of inner hair cells is associated with significant, or complete, loss of outer hair cells; in other words, outer hair cells tend to be the most vulnerable elements in the inner ear. In animal studies, one exception to this rule is the selective inner hair cell loss seen in the chinchilla after systemic administration of carboplatin, a chemotherapeutic drug (Takeno et al. 1994). In other species (e.g., guinea pig), carboplatin causes loss of both inner and outer hair cells (Taudy et al. 1992). Reasons for the unique response of the chinchilla cochlea to carboplatin remain unclear. However, it is interesting to consider a possible link to the thiamine-deprivation sensitivity of inner hair cells observed here. Case studies of platinum-based chemotherapy have described neurological symptoms, e.g., Wernicke's encephalopathy, characteristic of thiamine deficiency that can be reversed by thiamine supplementation (Kondo et al. 1996). An ameliorative effect of thiamine supplementation on platinuminduced hearing loss has not been investigated; however, other sulfur-containing compounds (e.g., methionine) can decrease the ototoxicity of carboplatin (Lockwood et al. 2000), presumably by formation of platinum-sulfur adducts that inactivate the platinum agents. Given that thiamine is a sulfur-containing compound, carboplatin's effects on inner hair cells may arise via interference with thiamine transport, either by thiamine binding or direct interaction with the high-affinity thiamine transporter studied here. To explain the differential platinum sensitivity of inner vs. outer hair cells in different mammalian species, one would have to postulate different distributions of the two thiamine transport systems (Slc19a2 and Slc19a3) in the two hair cell classes.

In human temporal bones, a pattern of selective inner hair cell loss has also been reported, but only in two studies (Amatuzzi et al. 2001; Slack et al. 1986); together, they suggest correlations with both premature birth and anemia. The more extensive of these studies evaluated patterns of cochlear histopathology in 30 ears from 15 patients who died after a stay in the neonatal intensive care unit. Of these 15 patients, eight showed no hair cell loss, two showed selective outer hair cell loss, two showed combined inner and outer hair cell loss, and three showed a bilateral pattern of selective inner hair cell loss similar to that seen here (Amatuzzi et al. 2001). The three patients with selective inner hair cell loss were all premature infants (30-32 weeks gestational age at birth), and only one other infant in the study was as premature. Correspondingly, all three of these innerhair-cell-affected patients also suffered from anemia, and only one of the remaining 12 shared this condition. The possible association between selective inner hair cell loss and premature birth is strengthened by a recent preliminary report suggesting a significantly increased risk of auditory neuropathy in babies born at or before 28 weeks gestation (Iwamoto et al. 2006). Recalling that human diseases associated with mutations in the SLC19A2 gene include hearing loss and anemia suggests that a way to link the human results with the present results is to hypothesize that (1) the high-affinity thiamine transporter is not adequately expressed in a premature infant at $\sim 30$ weeks gestational age, and (2) that this late expression elicits the same selective thiamine deprivation in inner hair cells as seen with absence of the transporter gene (as studied here). 


\section{ACKNOWLEDGMENTS}

This research was supported by grants (RO1 DC 04820 and P30 DC05209) from the NIDCD.

\section{REFERENCES}

Amatuzzi MG, Northrop C, Liberman MC, Thornton A, Halpin C, Herrmann B, Pinto LE, Saenz A, Carranza A, Eavey RD. Selective inner hair cell loss in premature infants and cochlea pathological patterns from neonatal intensive care unit autopsies. Arch. Otolaryngol. Head Neck Surg. 127:629-636, 2001.

Bertram S, Hoidis S, Smolders JWT, Gelb B, Oishi K, Mueller M. Critical role of thiamine transporter THTR1 in auditory function and hair cell survival. Association for Research in Otolarynoglogy Abstracts XXIXth Midwinter Meeting: 300, 2006.

Diaz GA, Banikazemi M, Oishi K, Desnick RJ, Gelb BD. Mutations in a new gene encoding a thiamine transporter cause thiamineresponsive megaloblastic anaemia syndrome. Nat. Genet. 22:309-312, 1999.

Fleming JC, Tartaglini E, Steinkamp MP, Schorderet DF, Cohen N, NeufELD EJ. The gene mutated in thiamine-responsive anaemia with diabetes and deafness (TRMA) encodes a functional thiamine transporter. Nat. Genet. 22:305-308, 1999.

Fleming JC, Steinkamp MP, Kawatsuji R, Tartaglini E, Pinkus JL, Pinkus GS, Fleming MD, Neufeld EJ. Characterization of a murine high-affinity thiamine transporter, Slc19a2. Mol. Genet. Metab. 74:273-280, 2001.

Fleming JC, Tartaglini E, Kawatsuji R, Yao D, Fujiwara Y, Bednarski JJ, Fleming MD, Neufeld EJ. Male infertility and thiaminedependent erythroid hypoplasia in mice lacking thiamine transporter Slc19a2. Mol. Genet. Metab. 80:234-241, 2003.

Huizing EH, de GRoot JC. Human cochlear pathology in aminoglycoside ototoxicity-a review. Acta Otolaryngol. Suppl 436:117-125, 1987.

Iwamoto L, Xoinis K, Weirather Y, Mavoon H, Shaha S. Extremely low gestation infants are at high risk for auditory neuropathy. Association for Research in Otolaryngology Abstracts XXIXth Midwinter Meeting: 72, 2006.
Kondo K, Fujiwara M, Murase M, Kodera Y, Akiyama S, Ito K, Takagi H. Severe acute metabolic acidosis and Wernicke's encephalopathy following chemotherapy with 5-fluorouracil and cisplatin: case report and review of the literature. Jpn. J. Clin. Oncol. 26:234-236, 1996.

Labay V, Raz T, Baron D, Mandel H, Williams H, Barrett T, Szargel R, McDonald L, Shalata A, Nosaka K, Gregory S, Cohen N. Mutations in SLC19A2 cause thiamine-responsive megaloblastic anaemia associated with diabetes mellitus and deafness. Nat. Genet. 22:300-304, 1999

Liberman MC, KIANG NYS. Acoustic trauma in cats, cochlear pathology and auditory-nerve activity. Acta Otolaryngol. 358:5-63, 1978.

Liberman MC, CHesney CP, KuJAwa SG. Effects of selective inner hair cell loss on DPOAE and CAP in carboplatin-treated chinchillas. Audit. Neurosci. 3:255-268, 1997.

Liberman MC, Gao J, He DZ, Wu X, Jia S, Zuo J. Prestin is required for electromotility of the outer hair cell and for the cochlear amplifier. Nature 419:300-304, 2002.

Lockwood DS, Ding DL, WANG J, SALVI RJ. D-Methionine attenuates inner hair cell loss in carboplatin-treated chinchillas. Audiol. Neuro-otol. 5:263-266, 2000.

Muller M, von Hunerbein K, Hoidis S, Smolders JW. A physiological place-frequency map of the cochlea in the CBA/J mouse. Hear. Res. 202:63-73, 2005.

Oishi K, Hofmann S, Diaz GA, Brown T, Manwani D, Ng L, Young R, Vlassara H, Ioannou YA, Forrest D, Gelb BD. Targeted disruption of Slc19a2, the gene encoding the high-affinity thiamin transporter Thtr-1, causes diabetes mellitus, sensorineural deafness and megaloblastosis in mice. Hum. Mol. Genet. 11:2951-2960, 2002.

Schuknecht HF. Pathology of the Ear. Harvard University Press, Cambridge, 1974.

Slack RW, Wright A, Michaels L, Frohlich SA. Inner hair cell loss and intracochlear clot in the preterm infant. Clin. Otolaryngol. Allied Sci. 11:443-446, 1986.

Starr A, Picton TW, Sininger Y, Hood LJ, Berlin Ci. Auditory neuropathy. Brain 119(Pt 3):741-753, 1996.

Takeno S, Harrison RV, Ibrahim D, Wake M, Mount RJ. Cochlear function after selective inner hair cell degeneration induced by carboplatin. Hear. Res. 75:93-102, 1994.

Taudy M, Syka, Popelar, Ulehlova. Carboplatin and cisplatin ototoxicity in guinea pigs. Audiology 31:293-299, 1992. 